\title{
Características coincidentes de estudiantes de Periodismo con talento en la escritura académica
}

\section{Matching characteristics in students of journalism with skills for academic writing}

DOI: https://doi.org/10.32870/dse.v0i23.858

Óscar Martín Vázquez Reyes*

Diana Irasema Cervantes Arreola**

Thelma Jovita García***

Dalia Lizette Gómez Martínez ${ }^{* * * *}$

\begin{abstract}
Resumen
El talento en la escritura académica de jóvenes universitarios es un tema poco explorado. Este estudio genera un acercamiento al perfil de estudiantes de Periodismo con dicho talento, basado en sus características comunes personales y de escritura, situado en los catalizadores ambientales e intrapersonales del Modelo Diferenciado de Dotación y Talento de Gagné, así como en algunos recursos del proceso de su desarrollo. Se llevó a cabo con un diseño de estudio de casos múltiple, con seis estudiantes del Programa de Periodismo. Los resultados arrojan varias coincidencias en las características de los catalizadores ambientales e intrapersonales, como alto desempeño académico, participación en concursos de redacción, ausencia de patrones para estudiar, poca sociabilidad, presencia de profesores detonantes del interés en la lectura y la redacción, pares retroalimentadores y motivantes, y prácticamente nula tradición lectora y escritural en la familia; los estudiantes se perciben como hábiles en motricidad fina, perfeccionistas, perseverantes y con el respeto como valor central. Se concluye que esas coincidencias en los casos son útiles para la construcción de una primera aproximación a un perfil de estudiantes de Periodismo con talento en la escritura académica.
\end{abstract}

Palabras clave: escritura - redacción - perfil del alumno - talento - periodismo.

* Maestro en Educación con especialidad en Procesos de Enseñanza-Aprendizaje. Profesor investigador de tiempo completo, Departamento de Humanidades, Programa de la Licenciatura en Periodismo. Universidad Autónoma de Ciudad Juárez. Chiuhuahua. México. oscar.vazquez@uacj.mx

** Doctora en Psicología. Miembro del Sistema Nacional de Investigadores. Líneas de investigación: aptitudes sobresalientes y talento en jóvenes, talento docente y procesos de enseñanza-aprendizaje. Profesora investigadora de tiempo completo, Departamento de Humanidades, Programa de la Licenciatura en Educación. Universidad Autónoma de Ciudad Juárez. Chiuhuahua. México. diana.cervantes@uacj.mx

*** Doctora en Educación. Doctora en Pedagogía. Profesora investigadora de tiempo completo, Departamento de Humanidades, Programa de Licenciatura en Educación. Universidad Autónoma de Ciudad Juárez. Chiuhuahua. México. thelma.garcia@uacj.mx

**** Maestra en Estudios Literarios. Profesora investigadora de tiempo completo, Departamento de Humanidades, Programa de la Licenciatura en Educación. Universidad Autónoma de Ciudad Juárez. Chiuhuahua. México.dalia.gomez@uacj.mx 


\begin{abstract}
Skilled academic writing in young university students is a seldom explored topic. This study generates an approach to the profile of journalism students with this skill, based on their common personal and writing characteristics, situated in the environmental and intrapersonal catalysts of the Gagné Differentiated Model of Giftedness and Talent, as well as some resources of the development process of this model. The study was conducted with a multiple case study design, with six students from the Journalism program. The results show several coincidences in the characteristics of the environmental and intrapersonal catalysts, such as high academic performance, participation in writing contests, absence of studying patterns, low sociability, the influence of teachers triggering reading and writing interest and of peers who motivate and give feedback, and practically no family tradition of reading and writing. The students perceive themselves as possessing fine motor skills, being perfectionist and persistent, and regarding respect as a core value. We concluded that these coincidences in the cases are useful for the construction of a first approach to a profile of Journalism students skilled in academic writing.
\end{abstract}

Key words: writing - drafting - student profile - talent - journalism.

\title{
Introducción
}

Durante la trayectoria académica de todo estudiante existen diversos elementos que lo apoyan a formar y construir habilidades; entre esos aprendizajes que se espera hayan desarrollado, de acuerdo con estudiosos del área (Carlino, 2003), se encuentran los referentes a la lectura y escritura académicas, encaminados al análisis y la producción con el dominio de un lenguaje academizado (alfabetización académica). Se pensaría entonces que cualquier joven, al ingresar a la educación superior, en ese sentido tendría un perfil homogéneo dadas las características de su formación escolar; sin embargo, el problema es que en realidad los estudiantes han tenido aprendizajes y experiencias académicas heterogéneas (Arcanio, Falavigna, Soler, 2013), por lo que sus habilidades y aptitudes son desiguales y plantean un reto mayor cuando entran al campo universitario.

En muchas ocasiones, las expectativas de agentes universitarios en torno a las habilidades de lectura y escritura son totalmente distintas a la realidad presentada por los alumnos, pues los estudiantes universitarios, de acuerdo con Roldán, Vázquez y Rivarosa (2011), enfrentan mayormente dificultades para resolver tareas que demandan leer y escribir, y Carlino (2003) advierte que el proceso de aprendizaje de escribir no se ha terminado al llegar al nivel superior, a pesar de que resulta sumamente necesario para cursar y aprender cualquier materia. En general, se plantea la escritura académica como una necesidad transversal que debe trabajarse en todos los niveles educativos, pues es importante su práctica y aprendizaje a lo largo del trayecto académico (Escalante-Barreto, 2015).

Y si bien, como queda claro, existen estudiantes con insuficientes habilidades para la escritura, también los hay que muestran capacidades y habilidades superiores en relación con sus pares etarios; en estos casos se está frente a jóvenes talentosos en la escritura académica. Ante 
este panorama, es importante plantearse de qué manera se puede potenciar la habilidad en la escritura académica en los espacios universitarios a partir de la experiencia y características de los alumnos con talento.

Marland (1971), Flanagan y Arancibia (2005), refieren que el talento académico tiene que ver no solo con acumular contenido académico, sino con cómo el individuo lo procesa. Sin embargo, en general hablar sobre talento implica enfrentarse a escasa literatura reciente y a las diversas concepciones, pues no existe acuerdo terminológico, y menos uno específico para el ámbito de la escritura (Pérez, López, González, 2008), por lo que no está claro cuáles serían las características o competencias para los casos de talento en la escritura académica; encontrarlas y describirlas contribuye a evidenciar los rasgos comunes de estudiantes en las áreas de redacción, así como al desarrollo de un posible perfil del talento de ese tipo.

\section{El talento académico}

Para conceptualizar el talento en la escritura académica es importante situar en primera instancia qué es el talento académico, ya que existen diferencias entre este y la aptitud sobresaliente con la que suele ser confundido.

Según Gagné (2008), el talento es el dominio destacado que de manera sistemática desarrolla capacidades, denominadas competencias (conocimientos y destrezas), que aparecen en al menos un área de la actividad humana, de tal manera que sitúa a la persona dentro del $10 \%$ superior a sus pares etarios activos en esa área. En su Modelo Diferenciado de Dotación y Talento, Gagné (2015) menciona que existen varios tipos de talento, entre ellos el académico, en el que se destacan competencias en el lenguaje, las ciencias, las matemáticas, lo vocacional y las humanidades. Describe que el apoyo a estudiantes con esas competencias en su área (por ejemplo, en la escritura) resulta fundamental para que se dé el proceso de desarrollo, por lo que es necesario conscientizarlos de que la lectura, y en particular la escritura, no son procesos terminados, sino en constante desarrollo a lo largo de la vida académica y personal, ya que el talento que no se desarrolla, se pierde (Gagné, 2015). En su modelo, también refiere que existen catalizadores (ambientales e intrapersonales) y un proceso de desarrollo (actividades, inversión y progreso) que permiten que el talento emerja. El presente estudio se ha centrado en analizar esta última parte del modelo.

Sucede una situación singular, pues generalmente los estudios sobre el talento se centran en la etapa de la niñez, por lo que los jóvenes y adultos con talento en general o en la escritura académica han sido poco estudiados. Dadas las características del modelo de Gagné, el caso del talento en la escritura académica en el nivel superior representa un campo para indagar acerca de estos catalizadores, pues estas capacidades pueden ser identificadas a lo largo de las diversas etapas del desarrollo ya que no solo se encuentran en la infancia o niñez, sino que perduran hasta etapas adultas (Reig, Reig, 2013). 
La escritura es un pilar estratégico para que los jóvenes universitarios construyan todo conocimiento; sin embargo, existen carreras de educación superior que, según la descripción de características ideales de aspirantes a ingresar, contemplan estudiantes con un perfil centrado en la redacción, dadas las condiciones de su profesión; un ejemplo es la Licenciatura en Periodismo, en la que se requiere que los alumnos cuenten con capacidades escriturales y lectoras destacadas.

\section{Contexto del estudio}

La Licenciatura en Periodismo ofrecida por la Universidad Autónoma de Ciudad Juárez (UACJ) busca que el aspirante a estudiarla tenga "facilidad para la comunicación oral y escrita, lo que equivale a una excelente redacción" (Universidad Autónoma de Ciudad Juárez, s.f. a, párr. 2), y que el egresado sea "experto en el manejo del lenguaje oral y escrito" (Universidad Autónoma de Ciudad Juárez, s.f. b, párr. 4). Según su descripción oficial, la formación redactora es la columna vertebral de la carrera, pues "las materias de teoría y técnicas periodísticas inician con la redacción general, pasan por español avanzado y continúan a lo largo de los géneros periodísticos" (Universidad Autónoma de Ciudad Juárez, s.f. c, párr. 11). Es evidente, entonces, que para avanzar o garantizar en lo posible su permanencia en ella, los estudiantes deban contar con bases y nociones redactoras sólidas; de ahí que el programa pueda considerarse campo fértil para encontrar alumnos con talento en la escritura académica, fundamental para el área, ya que contribuye en gran medida al desarrollo de la habilidad de escritura periodística; ambas, la escritura académica y la periodística, comparten elementos, enunciados al inicio de este apartado y en el fundamento teórico mencionado.

Identificar estudiantes talentosos para la escritura académica y describir sus características, resulta especialmente importante en Periodismo, pero también genera un primer paso en la definición del perfil que pudiera tener un universitario con talento en la escritura. Incluso, las miras de esta investigación van más allá, pues abonan datos en el ámbito de los talentosos, poco analizado sobre todo en edades avanzadas posteriores a la infancia; autores como Galdó (2007) consideran que hay un número importante de talentos sin aprovechar en la adultez, porque nunca fueron detectados.

Es entonces que, desde la mirada de estos datos e información existente, el objetivo del estudio es encontrar características coincidentes en estudiantes de Periodismo con talento en la escritura académica, a través de la descripción de sus catalizadores comunes ambientales, intrapersonales y recursos para el proceso de desarrollo, para empezar a conformar un perfil del talento en la escritura académica. Se plantea esta pregunta de investigación: ¿Qué características comunes en sus catalizadores ambientales e intrapersonales y en los recursos del proceso de desarrollo, describen el perfil del talento en la escritura académica, en jóvenes universitarios estudiantes de Periodismo? 


\section{Metodología}

La presente investigación tiene un enfoque cualitativo, con un diseño de estudio de casos múltiple.

Los participantes fueron definidos a partir de una fase inicial en la que 24 estudiantes de Periodismo fueron nominados por sus maestros. De esos 24, 14 aceptaron la invitación para participar en la segunda fase. En la tercera fase, de 14 que realizaron un ejercicio de redacción, fueron seleccionados 6 que mostraron elementos del talento en la escritura, ya que cumplían con la mayoría de los criterios contemplados tanto en el instrumento de nominación como en la rúbrica. Estos casos se describen a continuación con sus datos generales, por medio de una clave para cuidar el anonimato:

La informante 1, con asignación de clave PTEA1 ( $\mathrm{P}=$ Participante, TEA = Talento en la Escritura Académica), es mujer, soltera, de 22 años, de ocupación estudiante y trabajadora, de nivel socioeconómico bajo.

La informante 2, con clave PTEA2, es mujer, soltera, de 22 años, de ocupación estudiante y trabajadora, de nivel socioeconómico medio.

La informante 3, con clave PTEA3, es mujer, soltera, de 20 años, de ocupación estudiante y trabajadora, de nivel socioeconómico medio alto.

El informante 4, con clave PTEA4, es hombre, soltero, de 30 años, de ocupación estudiante y trabajador, de nivel socioeconómico medio alto.

El informante 5, con clave PTEA5, es hombre, soltero, de 18 años, de ocupación estudiante, de nivel socioeconómico medio.

Por último, el informante 6, con clave PTEA6, es hombre, soltero, de 20 años, con ocupación estudiante, de nivel socioeconómico bajo.

\section{Técnicas de recolección de la información}

En la primera fase del trabajo de investigación se utilizó una lista de nominados proporcionada por profesores de la Licenciatura en Periodismo, en la cual se consideraron elementos de acuerdo con el talento en la escritura académica que los docentes percibían en estudiantes destacados en sus clases. El formato de nominación enuncia aspectos como el conocimiento y aplicación de las reglas del código escrito, tendencia a la perfección en la escritura, originalidad, noción clara de la estructura de un texto, manejo extraordinario del vocabulario académico y habilidad para la lectura interpretativa, claridad de pensamiento, reconocimiento de relaciones implícitas y comprensión de significados, alta capacidad de concentración, capacidad de observación, reflexión en torno a los temas estudiados y al propio aprendizaje, buena percepción de las relaciones causa-efecto, posesión de alta cantidad de información y capacidad de interconectarla. Esta lista de nominación fue elaborada por los investigadores a partir de las nociones sobre talento escritural y académico expuestas en la literatura (tomadas de teóricos como 
Betancourt y Valadez, 2012; Flanagan y Arancibia, 2005; Gallagher y Gallagher, 1994; Shore y Kanevsky, 1993).

En una segunda fase, se trabajó con un ejercicio de redacción para corroborar la percepción de los docentes en la nominación de sus alumnos. El ejercicio consistió en la realización de un texto narrativo y reflexivo de 2.5 cuartillas estandarizadas en el programa Word, sobre un tema de resonancia periodística muy reciente, sin duda conocido por los estudiantes. Para la valoración de los resultados del texto se diseñó una rúbrica con puntuaciones del 4 al 1 (de la máxima a la mínima) que consideraba los parámetros de la lista de nominación docente, así como los rubros de título, introducción, desarrollo y conclusión, coherencia, estructura gramatical, sintaxis, ortografía, puntuación, originalidad, uso de giros del lenguaje y de vocabulario/ léxico, conocimiento de la información, capacidad de observación y reflexión crítica. Antes de su aplicación, la rúbrica fue sometida a juicio de dos especialistas en lingüística, quienes la analizaron y comentaron.

En la tercera fase del trabajo de investigación, los seis estudiantes participaron en una entrevista a profundidad elaborada a partir del Modelo Integral de Desarrollo del Talento (CMTD, por sus siglas en inglés) de Gagné (2015). Con ella se buscó ahondar en los catalizadores que sirven como puente entre las capacidades naturales y el talento, por lo que se indagó sobre los ambientales (medio, individuos y servicios), y los intrapersonales (rasgos físicos y mentales, manejo de objetivos: de conciencia, motivación y voluntad) que pudieran arrojar indicios sobre las causas por las que cada individuo desarrolló un talento específico en la redacción.

La entrevista incluye 54 preguntas, divididas en dos grandes partes con respecto al modelo de Gagné (2015): rasgos ambientales del modelo y rasgos intrapersonales. La primera parte considera recursos como trayectoria académica y formación escolar (preguntas de la 1 a la 17), desarrollo personal (preguntas de la 18 a la 24), influencia de pares y maestros (preguntas de la 25 a la 27), autopercepción en torno a las dotes escriturales (preguntas de la 28 a la 33), y la influencia de individuos y elementos en el entorno familiar (preguntas de la 34 a la 42). La segunda parte contempla los rasgos físicos y mentales (preguntas 43 y 44), manejo de objetivos (preguntas 45 y 46), motivación (preguntas de la 47 a la 50) y voluntad, concepto y autoestima (preguntas de la 51 a la 54).

Todos los datos e información obtenidos en los instrumentos fueron resguardados para cuidar la confidencialidad y el anonimato de los participantes.

\section{Análisis de la información}

La información obtenida en la primera fase, fue analizada a partir de un concentrado en una hoja de cálculo de Excel, con los elementos de la lista de nominación, donde se agregaron los datos de cada participante; se hizo un cruce dada la nominación de los profesores y se constató que en las 24 nominaciones los maestros consideraron la gran mayoría de los elementos señala- 
dos. En la segunda fase, tres especialistas (dos profesionales de literatura y uno de periodismo) analizaron los textos narrativos y aplicaron y llenaron la rúbrica de valoración del ejercicio de redacción realizado por 14 alumnos que atendieron la invitación; posteriormente, se hizo un cruce de resultados para determinar quiénes tenían más elementos del talento en la escritura académica de acuerdo con dicha rúbrica. En la fase tres, de la entrevista realizada a los seis casos seleccionados, se examinó la información vaciada en una hoja de cálculo de Excel, bajo las categorías predeterminadas en la guía de entrevista a profundidad, y se hizo el entrecruce de similitudes y coincidencias.

\section{Resultados}

Las entrevistas permitieron encontrar puntos de coincidencia en características del talento en la escritura académica de los estudiantes de Periodismo analizados en la investigación. Este cruce de datos se centró en los catalizadores del Modelo Integral de Desarrollo del Talento de Gagné (2015), los cuales comprenden dos categorías centrales: catalizadores ambientales e intrapersonales. Los catalizadores ambientales se dividen en estas categorías: $a$ ) trayectoria y formación académica, $b$ ) desarrollo personal y c) individuos (profesores, mentores, familia, pares); los intrapersonales tienen las categorías a) rasgos físicos y mentales y $b$ ) manejo de objetivos; este último con las subcategorías b1) conciencia, b2) motivación y b3) voluntad, detonantes del desarrollo del talento en la escritura académica (figura 1). Para ambas categorías centrales también se exploró la categoría recursos, la cual sirvió para conocer el proceso de desarrollo del talento en la escritura académica, por lo que se considera transversal a todos los catalizadores. 
Figura 1. Categorías centrales: catalizadores ambientales e intrapersonales, con sus categorías y subcategorías prestablecidas a partir del Modelo Integral de Desarrollo del Talento de Gagné (2015)
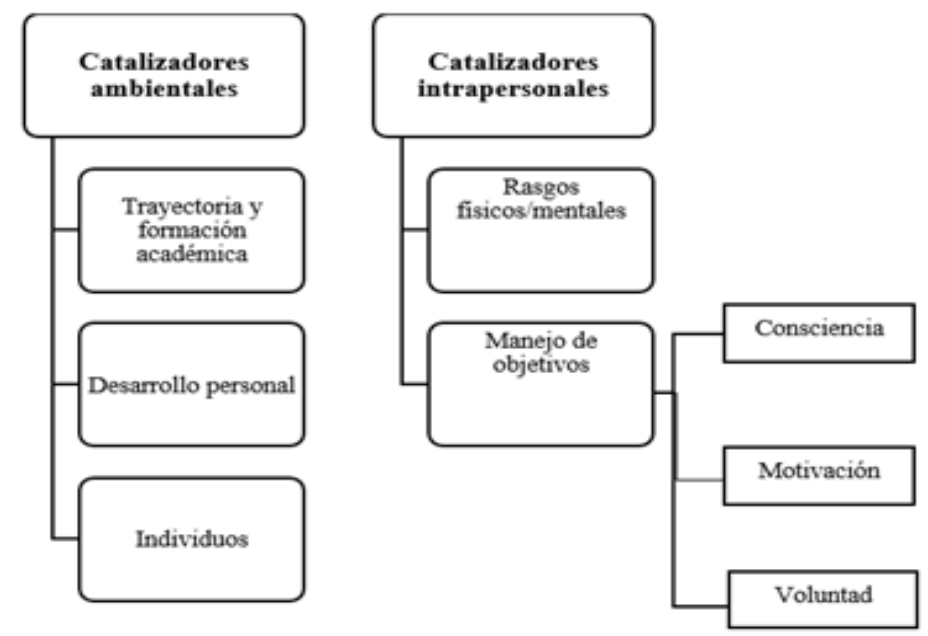

Proceso de desarrollo

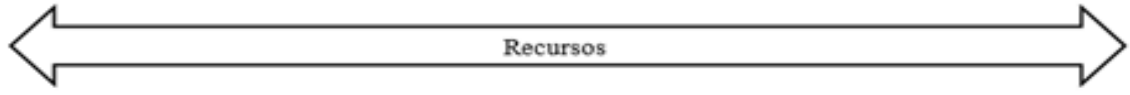

A continuación, se presentan los resultados obtenidos bajo las categorías de análisis predeterminadas.

\section{Catalizadores ambientales}

\section{a) Trayectoria y formación académica}

De los elementos destacados en la categoría de Trayectoria y Formación Académica, se define que PTEA1, PTEA2 y PTEA4 cursan el nivel avanzado de la Licenciatura en Periodismo y PTEA3, PTEA5 y PTEA6 se encuentran en un nivel intermedio. Los seis participantes asistieron al preescolar, y de estos PTEA2, PTEA3, PTEA4 y PTEA5 entraron directamente a tercer grado de kínder. PTEA2 y PTEA3 coinciden en que la etapa preescolar les enseñó a socializar, mientras que PTEA1, PTEA2 y PTEA5 no recuerdan cosas trascendentales respecto al aprendizaje académico o su estadía durante esa época. Tanto PTEA3 como PTEA4 mencionan que lo más notable que recuerdan son las cuestiones asociadas al juego; sin embargo, PTEA4, PTEA5 y PTEA6 refieren actividades preparatorias para la lectura o la escritura:

Recuerdo que las primeras tareas eran... o sea, hacer planas de ciertos signos, como palitos o casitas, yo tenía entendido que eso nos iba a ayudar posteriormente para escribir (PTEA5).

La parte dedicada a la enseñanza de la escritura y del leer, lectura. Es lo que más recuerdo de esa etapa, la verdad (PTEA6). 
Con respecto al periodo de primaria, PTEA1, PTEA2 y PTEA3 destacan su alto desempeño académico; PTEA1 y PTEA3 participaron reiteradamente en concursos académicos y coincidieron en el Concurso Estatal “Don Quijote nos invita a leer". Para PTEA3, PTEA4 y PTEA5 el interés por la lectura surgió en este periodo:

Fue hasta quinto grado de primaria cuando empecé a leer por gusto, empecé a leer libros de literatura un poco más de niños o juvenil (PTEA3).

Me gustaba mucho el de, el de lecturas, porque traía poemas, y luego lo acompañan, lo acompañaban con, con dibujos, y como que eso te ayudaba a, a imaginarte más cosas [...] ahí empecé a, a tener como más contacto con, con los textos, y a partir de ahí, siempre [...] a desarrollar como que el gusto por, por la lectura (PTEA4).

Hasta sexto de primaria, recuerdo que un profesor, uno de los mejores que me han enseñado, nos empezó a repartir libros, para que fuéramos leyéndolos y ya después [...] teníamos que pasar al frente y nos hacía preguntas del libro y teníamos que responder (PTEA5).

Cinco de los participantes (PTEA1, PTEA2, PTEA3, PTEA4 y PTEA5) señalan que en el nivel secundario todo era más relajado, que bajaron su desempeño académico y no tenían gran motivación por la lectura y la escritura en general. PTEA2, PTEA3, PTEA5 y PTEA6 coinciden en que volvieron a interesarse en la lectura y la escritura al final de la escuela secundaria:

Recuerdo que me gustaba mucho escribir, me gustaba mucho hacer mis propias historias, eh, fue cuando empecé a entrar un poquito más en romance, un poquito más en poesía [...] solo el último año me tocó compartir con compañeros libros un poquito más de adolescentes (PTEA3).

Mientras hacía escritos en tercer semestre [...] perdón, tercer año, eh, me di cuenta que se me daba un poco más la escritura (PTEA6).

En el nivel preparatoria los seis participantes se destacaban en lectura o escritura. PTEA2 y PTEA3 participaron activamente en un taller que fomentó la escritura y la lectura; coincidieron en la misma institución, pero en periodos distintos:

Pero lo que fue la constante fue siempre tener buenas calificaciones en lo que era en materia de literatura, de redacción, de español (PTEA1).

[...] primero entré a un círculo de lectura, así como a talleres de los fines de semana, y de ahí empecé a estar en concursos y en cosas así (PTEA2).

En la preparatoria pues continué escribiendo, empecé a leer, como, como ya estaba más de moda, empezaba a leer recomendaciones de mis amigas, este, de autores como John Green, ah... Verónica Roth, autores un poquito más estadunidenses y utilicé la lectura para, eh, para poder fortalecer mi inglés [...] 
seguí escribiendo por mi cuenta, empecé a escribir diarios [...] mis trabajos, a pulirlos más en ortografía [...] mi maestra de teatro era muy, este, perfeccionista con estas cuestiones de la acentuación, porque ella estudió lenguas (PTEA3).

Me metí a ese, a ese concurso de ensayo, estuve tallereándolo con una profesora de filosofía, no era muy buena, pero, este, ahí [...] ya mandé el trabajo y... y sí se seleccionó y... fuimos a una especie como de plática-congreso, donde platicamos de nuestro trabajo [...] empezaba a leer cuentos, me gustaba escribir, escribir cuentos, y de repente que veía una cosa interesante que veía en la calle, la anotaba (PTEA4). Y creo que fue hasta tercero, de tercer semestre de, de preparatoria, que volví a leer un libro completo [...] Me parece que ya para entonces ya había leído Drácula [...] y no sé cómo que empecé a buscar referencias literarias, porque había visto películas que tenían referencia con la literatura (PTEA5).

Con respecto a otras actividades creativas, durante el periodo escolar de la preparatoria, tanto PTEA1 y PTEA2 como PTEA4 han dibujado en algún momento de su vida:

Desde muy pequeña dibujaba, seguí recibiendo la formación en dibujo en la secundaria con el dibujo técnico y supongo que sí, eso me ayuda más a soltar, a soltarme, ¿no? (PTEA1).

Primero estuve en un... en un taller de dibujo y... ahí estaba más bien porque, pues había mucha gente que le gustaba el "animé" y todas esas cosas [...] duré ahí como medio año, nada más (PTEA2).

En tanto, PTEA3 y PTEA5 coinciden en su orientación hacia la pintura, mientras que PTEA3 y PTEA4 desarrollaron actividades en el ámbito teatral.

Tomaba clases de baile, tomaba clases de pintura (PTEA3).

Había un curso de dibujo para los fines de semana, en Bachilleres, los sábados, entré, me inscribí a ese curso, pero me di cuenta de que era más pintura que dibujo [...] fue cuando empecé a hacer mi primer, mi primer cuadro (PTEA5).

Los seis participantes estudiaron en escuelas públicas. Fue poca la oferta de cursos extracurriculares que no fueran académicos; solo PTEA3 y PTEA4 coincidieron en realizar actividades extracurriculares, pero no propias de sus escuelas.

En cuanto a su autopercepción como estudiantes, PTEA1, PTEA2 y PTEA3 se consideran buenos, es decir, dedicados al estudio; sin embargo, atravesaron una etapa de rebeldía:

Me he considerado una buena estudiante, no excelente, porque creo que siempre se puede dar más [...] yo estaba en una etapa rebelde (PTEA1).

Pues, en la primaria era muy recta, en la secundaria fue cuando empecé a decaer [...] Creo que también, no subí tanto como en la primaria, pero sí empecé, porque sí era más complicado (PTEA2). 
Creo que era buena estudiante, era un poco distraída porque siempre tenía muchas cosas que hacer y todavía, pero creo que también fui un poco rebelde (PTEA3).

De las materias que más les han gustado durante la formación preuniversitaria, cinco participantes coincidieron que Español (PTEA1, PTEA2, PTEA3, PTEA4 y PTEA5); PTEA6 la menciona como Redacción. Para PTEA2, PTEA4, PTEA5 y PTEA6, Biología también fue de sus predilectas; PTEA1, PTEA4 y PTEA5 señalan Literatura como otra de sus favoritas; PTEA2, PTEA3 y PTEA6 también Química. Otras de las preferidas en menor grado de coincidencia fueron Comunicación e Inglés. En cuanto a las materias que menos les gustaron, PTEA3, PTEA4, PTEA5 y PTEA6 coinciden en las relacionadas con los números (Matemáticas y Cálculo Integral), mientras PTEA1 y PTEA4, en Química.

En cuanto a la Licenciatura en Periodismo, PTEA1, PTEA2, PTEA4 y PTEA6 dicen haber elegido la carrera por su relación con la escritura o lo literario:

Por eso estudié periodismo y también porque está muy relacionada a la escritura y desde que estaba pequeña era lo que se me facilitaba. Yo desbordaba letras (risas) [...] escribir siempre estuvo ahí, lo que siempre fue algo por lo que destaqué (PTEA1).

[...] entonces me interesó mucho la corrección y, a raíz de eso, yo dije: "ah, pues estudio literatura o estudio periodismo" [...] el periodismo se me hizo que tenía como que más campo laboral (PTEA2).

Me gustaba mucho el trabajo que hacían los, los reporteros, cuando los veía en, en televisión, o cuando leía, o sea [...] también leía el periódico y estas cosas, y me veía yo [...] entonces decía: "eso es lo que me gustaría hacer", y veía que también lo podía combinar con la literatura, y hay periodistas que también son escritores, y como que también van de la mano (PTEA4).

Porque se me da la escritura (PTEA6).

Para PTEA3 y PTEA5 su elección se relaciona más con su interés por estudiar Ciencias de la Comunicación; sin embargo, decidieron ingresar a Periodismo por razones relacionadas con la escritura y el manejo de los medios de comunicación.

En cuanto a las materias que más les han gustado durante la licenciatura, los seis participantes coinciden en Historia; PTEA1, PTEA2, PTEA3 y PTEA5, en Redacción; PTEA1, PTEA3 y PTEA4, en Fotografía, y PTEA1, PTEA2 y PTEA3, en Sociología. Entre las materias que menos les han gustado, PTEA1, PTEA3 y PTEA6 mencionan Lógica; PTEA2 y PTEA3, Televisión y Radio, y PTEA3 y PTEA6, Códigos Deontológicos.

Sobre las notas que generalmente han obtenido en las materias que más les han gustado a lo largo de su vida académica, PTEA1, PTEA4 y PTEA5 coinciden en una calificación arriba de 8; para PTEA2, PTEA3 y PTEA6, es 9, arriba de 9 o 10.

Cuando se indagó acerca de las prácticas de estudio, PTEA1, PTEA2, PTEA4, PTEA5 y PTEA6 consideran que no siguen ningún método, pero PTEA1, PTEA2 y PTEA4 leen y usan la memorización como parte del estudio, y PTEA3 y PTEA4 consideran tomar notas: 
Pues, estar leyendo y leyendo, y dándole hasta que ya se me queda o entiendo bien (PTEA2).

Por lo regular, tiene que ser leer [...] casi siempre este método de lectura utilizo (PTEA4).

En cuanto a los cursos extracurriculares, PTEA1, PTEA2, PTEA3 y PTEA4 han realizado por los menos una actividad extracurricular en su historia de vida. PTEA1 y PTEA3 coinciden en cursos de pintura; PTEA2 y PTEA3, cursos de baile (danza moderna, baile contemporáneo, ballet, hip hop) y alguna actividad relacionada con el deporte (natación, básquetbol y voleibol), y PTEA3 y PTEA4, cursos de inglés.

En la figura 2 se presenta el cruce de las coincidencias del análisis de esta categoría.

Figura 2. Cruce de coincidencias de la categoría Trayectoria y Formación Académica

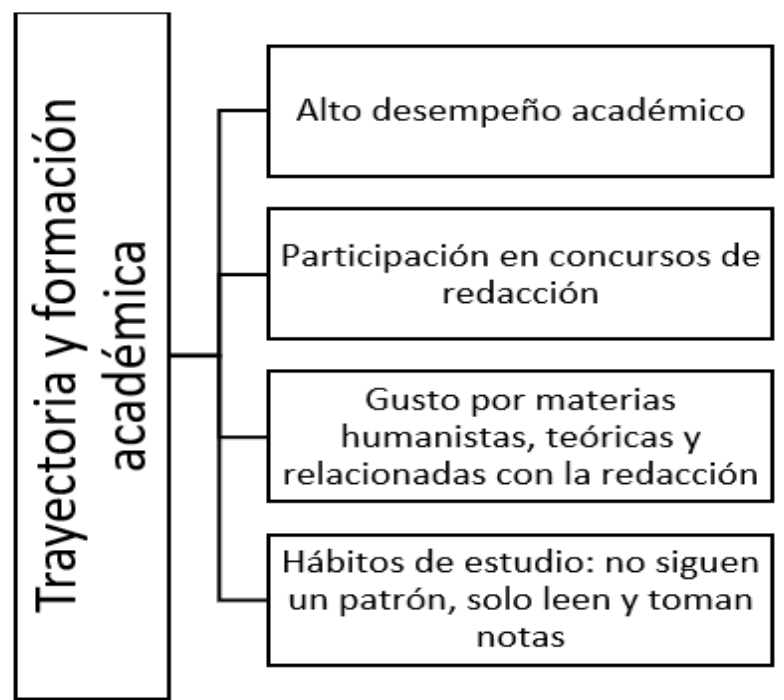

\section{b) Desarrollo personal}

En cuanto a las coincidencias que se encontraron en la categoría de Desarrollo Personal, relacionadas con sus pasatiempos y actividades en su tiempo libre, a PTEA1, PTEA2, PTEA3, PTEA5 y PTEA6 les gusta ver películas y series; PTEA2, PTEA4, PTEA5 y PTEA6 escuchan música; PTEA2, PTEA3 y PTEA4 leen; solo PTEA3 y PTEA4 escriben.

Respecto a las actividades extracadémicas, políticas y culturales, PTEA3 y PTEA5 realizan labores para revistas en línea (edición y redacción de artículos). Y si bien algunos de los participantes no consideran que su vida social sea mala, PTEA2, PTEA3, PTEA4, PTEA5 y PTEA6 expresan que muchas de sus interrelaciones son escolares y prefieren no salir ni socializar mucho: 
Regularmente [...] yo no me acerco, la gente se acerca a mí (PTEA2).

Yo creo que sí tengo una vida social bastante buena en comparación del pasado, que me cuesta relacionarme, sí, un poco todavía, porque pues, me da mucha ansiedad a veces, eh, las cuestiones sociales, relacionarte, seguir una conversación fuera de lo personal (PTEA3).

Un poco reservado, o sea, en cuestiones como para confiar cosas más personales [...] ahora no, ahora no tanto, sí salgo, pero así, contadas ocasiones (PTEA4)

Tiendo, tiendo a socializar más cuando estamos en periodos académicos, cuando estamos en clases [...] fuera de las clases raramente socializo con ellos [...] sigo teniendo esta idea de que mi casa es mucho más cómodo que estar afuera (PTEA5).

Eh, ¿amigos, todo eso? Bueno, puede decirse que suelo ver a mis amigos en ciertas actividades, pero no, no acostumbro a salir a ningún lado con ellos [...] Eh, para qué le miento, eh, meramente escolares, casi siempre (PTEA6).

En torno a otro aspecto relacionado con el desarrollo personal, el hábito de la lectura, PTEA2, PTEA3 y PTEA4 leen de manera constante, principalmente temas relacionados con el periodismo; PTEA1, PTEA5 y PTEA6 lo hacen de manera poco regular. Respecto a los hábitos de escritura, PTEA2, PTEA4, PTEA5 y PTEA6 refieren que no escriben de manera constante y solo lo hacen cuando es obligatorio para fines académicos; PTEA1 y PTEA2 escriben constantemente y coinciden en llevar a cabo escritura creativa como la poesía.

Me gusta escribir cuando algo me mueve, me gusta la poesía en prosa, los cuentos se me hacen muy difíciles, he intentado escribir cuentos, pero no lo he logrado, entonces me quedo más con la poesía en prosa y la crónica (PTEA1).

Sí, sí me gusta escribir, ah, tengo un proyecto en redes sociales que, que pues nació... pues de muchos cambios en mi vida [...] y luego tomé la clase de literatura contemporánea, entonces fue como una manera de trabajarlo, me gusta escribir, pues... como un poquito más de poesía alternativa (PTEA3).

En figura 3, se resumen las coincidencias en la categoría de Desarrollo Personal. 
Figura 3. Cruce de coincidencias de la categoría Desarrollo Personal

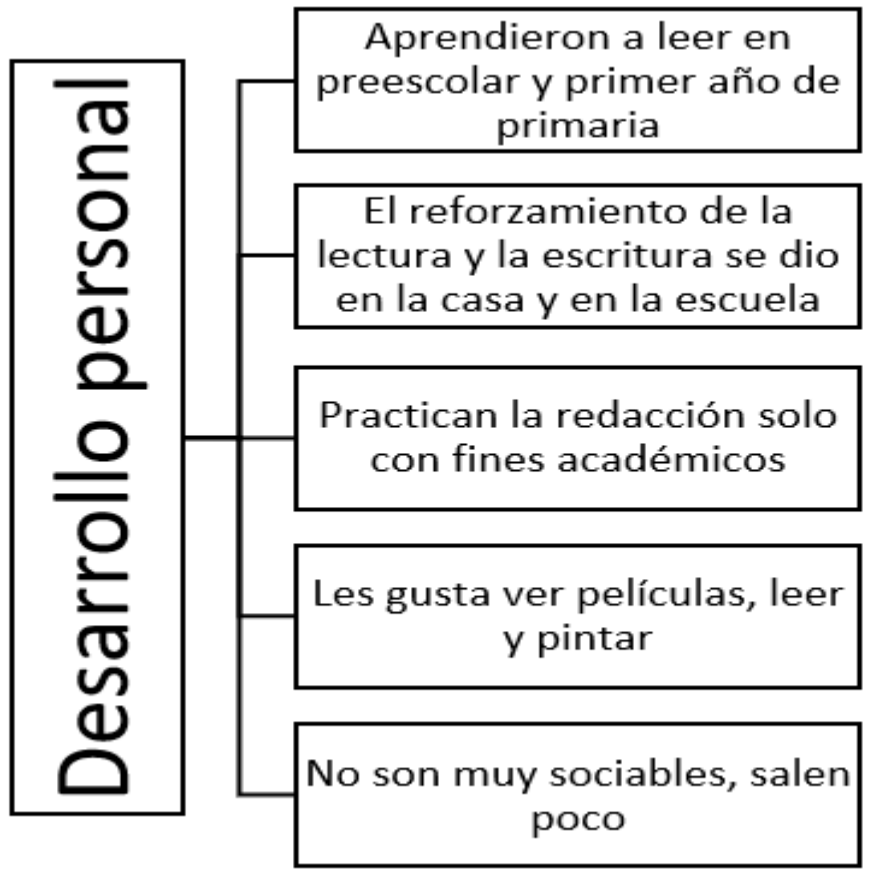

\section{c) Individuos}

En la categoría Individuos (profesores), para PTEA1, PTEA4, PTEA5 y PTEA6, a lo largo de su historia académica los docentes han sido significativos en el desarrollo de sus habilidades de lectura y escritura; para PTEA3, lo han sido en el nivel universitario.

En cuanto a las acciones de los profesores para detonar el interés por la redacción y la lectura, PTEA1, PTEA4 y PTEA5 coinciden en la recomendación de libros y lecturas como apoyo para su formación en habilidades de lectura y escritura:

Ella también era muy abierta en esos aspectos y eso hizo que me interesara más en ese ámbito, y nos daba libros, y nos recomendaba libros de acuerdo con el perfil [...] ya en la universidad, muchos profesores nos recomiendan muchos libros y pues gran parte de esos nos interesan por la carrera (PTEA1). Las lecturas que a veces nos ponían a hacer, o sea, nos hacían lecturas sugeridas [...] de ciertos autores y ya tú elegías el que más te interesara (PTEA4).

La recomendación de libros de texto, de libros de literatura, la recomendación, ya a nivel universitario [...] por eso yo creo que esos han sido los detonantes para darme cuenta de que, primero, tengo problemas de redacción, y luego, que puedo mejorar (PTEA5).

PTEA2, PTEA3 y PTEA6 consideran que una acción de sus profesores, detonante del interés por la redacción y la lectura, ha sido la revisión minuciosa, específicamente en el nivel universitario: 
Son muy meticulosos con lo que les entregamos, y eso provoca que uno [...] mismo se vaya corrigiendo los errores que tiene (PTEA2).

[...] tuvimos una clase donde eran mucho más estrictos, si tenías un error, por ejemplo, de ortografía o de redacción de síntesis, de sintaxis, em, pues tu trabajo se invalidaba [...] te daban otras dos oportunidades, después de la tercera oportunidad se invalidaba totalmente el trabajo, y era un poco de presión [...] pero te obligaba a hacer una revisión exhaustiva de tu trabajo (PTEA3).

En la categoría Individuos (pares), PTEA1, PTEA3, PTEA4, PTEA5 y PTEA6 consideran que los compañeros y amigos sí han influido de manera positiva en el desarrollo de sus habilidades de escritura, por la retroalimentación, por motivación o por la sana competencia:

Sí han ayudado bastante, mucho... Siento que no les duele a veces decirte cuando algo está mal, ¿no?, y eso ayuda bastante [...] te dicen cosas como: "oye, aquí está mal", dando tips [...] son críticos, incluso muchos de mis amigos ya egresaron, pero seguimos teniendo contacto y a veces hacemos talleres y llevamos textos y los leemos entre todos y hacemos crítica (PTEA1).

Antes, yo creo que influían en que me pedían ayuda y no quería que sacaran malas notas por mí si yo les ayudaba y me equivocaba, creo que eso no me gustaba, entonces, si no sabía algo, o no estaba segura, me motivaba a buscar qué era lo correcto para poderle ayudar a la persona (PTEA3).

Es una competencia, no como envidia, vamos, era algo así como decir, pues yo también lo puedo lograr (PTEA4).

Mis amigos de la universidad, yo creo que bastante, porque, no sé, yo creo que ellos se han formado una imagen de mí [...] no sé, siento que eso me da una motivación para, no sé si cumplir o hacer realidad la imagen que tienen de mí (PTEA5).

Normalmente me decían, no... me dicen, "eh, escribes bien, eh..." Sí me hacían esos comentarios constantemente (PTEA6).

En la categoría Individuos (familia) destaca que cuatro de los participantes coinciden en un entorno económico de clase media baja; los otros dos refieren un nivel socioeconómico bajo. Los integrantes de sus familias, tanto padres como hermanos, son trabajadores en diversas ramas; solo los padres de dos de ellos cuentan con negocio propio.

En cuanto a los estudios de los padres de los participantes, se refiere que algunos han estudiado hasta secundaria y preparatoria; en los casos de PTEA2, PTEA3, PTEA4 y PTEA5 uno de los dos padres cuenta con estudios de licenciatura. PTEA2, PTEA3 y PTEA5 tienen hermanos que estudiaron o están estudiando en la universidad.

Analizando estos datos, se trasluce que sus habilidades en la escritura no necesariamente tienen una relación con el grado de estudios de sus familiares, además de que no existe tradición lectora o de escritura en la familia. Respecto al nivel socioeconómico en el que se han 
desarrollado los seis participantes, este parece no influir con el desarrollo de sus competencias para el talento en la escritura académica.

En relación con la edad en que aprendieron a leer y escribir, PTEA1 y PTEA3 refieren que fue antes de entrar a primaria: "Todavía estaba más o menos por el kínder, iba saliendo del kínder, apenas iba a entrar a la primaria" (PTEA1). PTEA2, PTEA4, PTEA5 y PTEA6 mencionan que aprendieron en la primaria: "Creo que como a los... 6, 7, pues, en ese lapso de primer y segundo grado de primaria" (PTEA2). "Cuando entré a la primaria" (PTEA4). PTEA1, PTEA2, PTEA4, PTEA5 y PTEA6 aprendieron a leer y escribir en la escuela. PTEA3, PTEA4 y PTEA5 mencionan que, en su casa, sus padres o hermanos les leían: “Yo creo que mi mamá me llegó a leer cuando estaba en el proceso de aprender" (PTEA3). "En primero de primaria nos daban un libro de lecturas de cuentos, eh, entonces mi hermana me leía antes de dormir" (PTEA5).

PTEA3, PTEA5 y PTEA6 recuerdan que sus padres los ponían a leer, principalmente cuentos, la mayor parte del tiempo con fines académicos: "Me mandaron a leer, y mucho este análisis de '¿qué entendiste, y qué decía la lectura, y quién es el personaje?', y esas cosas [...] cuentos de Disney, luego yo sola empecé a pedirles que compraran estos libritos que se llaman Selecciones" (PTEA3).

PTEA1, PTEA4 y PTEA6 refieren que de pequeños les gustaba escribir:

Me gustaba mucho escribir cuando estaba muy niña, de hecho, actualmente ya no escribo tanto, pero cuando era pequeña sí escribía mucho, mucho, mucho, sobre lo que veía, sobre lo que soñaba [...], cuando era pequeña me gustaba mucho hacer rima, los versos, y leerlos en la escuela, y al salón (PTEA1). Sí, sí me gustaba [...] no escribía como que cosas acá muy literarias, pero sí escribía, me ponía a hacer, a copiar [...] si veía un cuento o algo, o algún poema, luego la pasaba a mi libreta [...] mmm, me gustaba mucho lo de la cuestión de la caligrafía, entonces, siempre buscaba como la manera de mejorar las letras, de mi forma de escribir, me compraban muchas libretas de doble raya, y me gustaba mucho escribir en esas libretas (PTEA4).

A PTEA2 y PTEA6 sus padres los ponían a escribir; sin embargo, esta práctica estaba relacionada con cuestiones meramente académicas.

La figura 4 presenta las coincidencias sobre varias características en la categoría Individuos. 
Figura 4. Cruce de coincidencias en la categoría Individuos

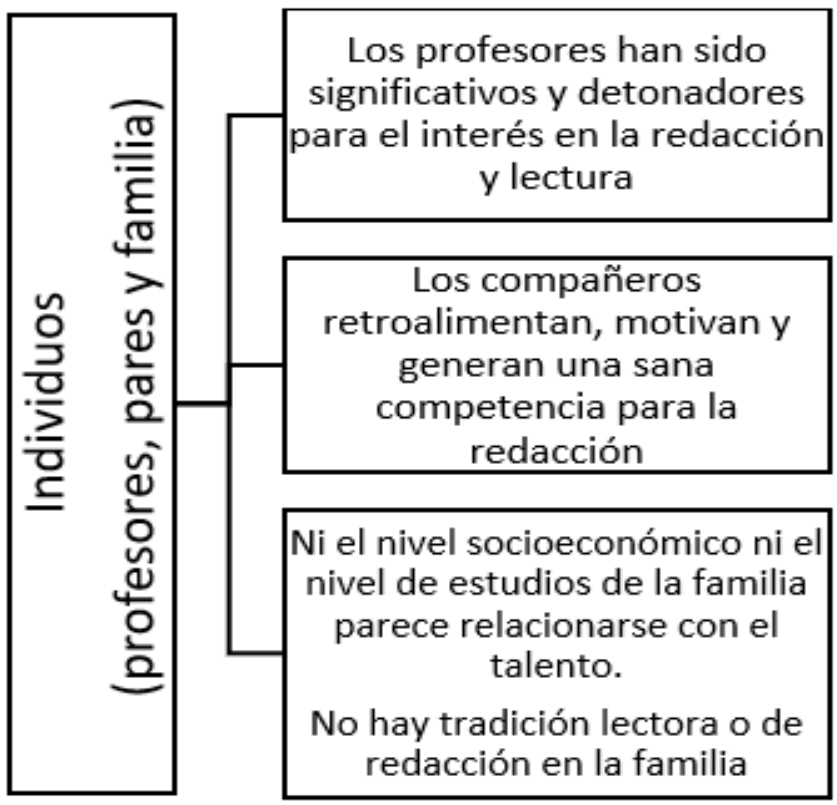

\section{Catalizadores intrapersonales}

\section{a) Rasgos físicos y mentales}

En cuanto a sus rasgos físicos, los seis participantes coinciden en que no presentan problemas motrices que afecten su desarrollo personal o académico. Manifiestan habilidades y motricidad fina, como buen balance físico y habilidad manual. Al respecto solo PTEA2 menciona que además de ser ambidiestra tiene miopía y astigmatismo.

Balance físico (PTEA1).

Cierta velocidad para escribir, habilidad con las manos (PTEA3).

Buena vista (PTEA5).

El oído y la vista, escribir manualmente (PTEA6).

En referencia a su personalidad, 50\% de los entrevistados se asumen introvertidos o tímidos:

Soy muy introvertida, soy muy cerrada (PTEA2).

Soy una persona reservada (PTEA4).

Yo creo que el ser retraído, tímido me ha ayudado a expandir, este, mis habilidades de escritura (PTEA5).

Dos de los seis entrevistados se consideran empáticos. PTEA6 es el único que menciona ser comprometido y responsable: 
Soy muy introvertida [...] Evito el conflicto, soy empática, paciente (PTEA2).

Soy empática (PTEA3).

Soy responsable, un poco desordenado, puntual, comprometido (PTEA6).

PTEA1, PTEA3, PTEA4 y PTEA6 consideran tener buena autoestima; PTEA2 y PTEA5 dicen que su autoestima es baja:

Siento que tengo buena autoestima [...] Me he aceptado (PTEA1).

Creo que es muy buena, por esta cuestión que la he ido trabajando, creo que nunca tuve como una autoestima baja (PTEA3).

Considero que es alta (PTEA6).

En la figura 5 se muestran las coincidencias detectadas en el análisis de esta categoría.

Figura 5. Cruce de coincidencias en la categoría Rasgos Físicos y Mentales

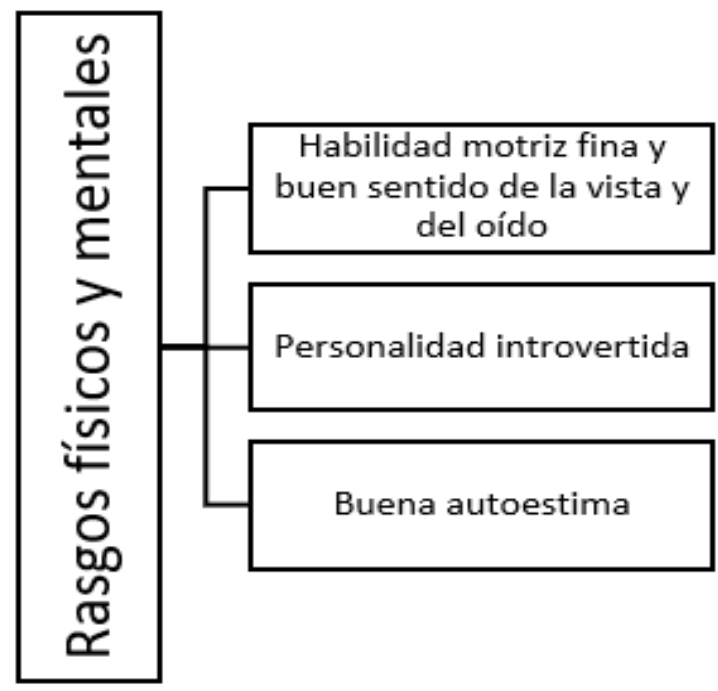

\section{b) Manejo de objetivos}

En la subcategoría de Consciencia, PTEA2, PTEA3, PTEA4 y PTEA5 destacan que se asumen en sus fortalezas como perfeccionistas; PTEA1 y PTEA6 subrayan ser tenaces:

Soy una persona muy dedicada, me gusta hacer las cosas bien (PTEA1).

Creo que mi visión artística, mi pasión y sensibilidad social, liberal, curiosa [...] Soy paciente (PTEA3).

Nunca estoy satisfecho con nada de lo que hago, lo cual me motiva a escribir bien (PTEA5). 


\section{b 1) Conciencia}

En torno a la conciencia de sí mismos (b1), en el autocontrol, PTEA1, PTEA2, PTEA3, PTEA4 y PTEA5 refieren que en circunstancias difíciles no se autocontrolan; sin embargo, PTEA6 dice sí apelar al raciocinio para autocontrolarse.

Sobre sus debilidades, PTEA1, PTEA5 y PTEA6 mencionan que tienden a procrastinar, mientras que PTEA2 y PTEA4 se consideran indecisos y poco organizados:

Dejo pasar mucho tiempo para hacerlas (actividades), no me organizo bien (PTEA2).

Soy muy emocional [...] distraída (PTEA3).

En ocasiones no soy constante... indeciso (PTEA4).

Tiendo a dejar actividades, ah, que me interesan para después (PTEA5).

En todos los casos aparece como un factor común el postergar o el pensar demasiado sobre lo que se tiene que hacer.

La figura 6 muestra las coincidencias obtenidas en esta subcategoría.

Figura 6. Cruce de coincidencias en la subcategoría Consciencia

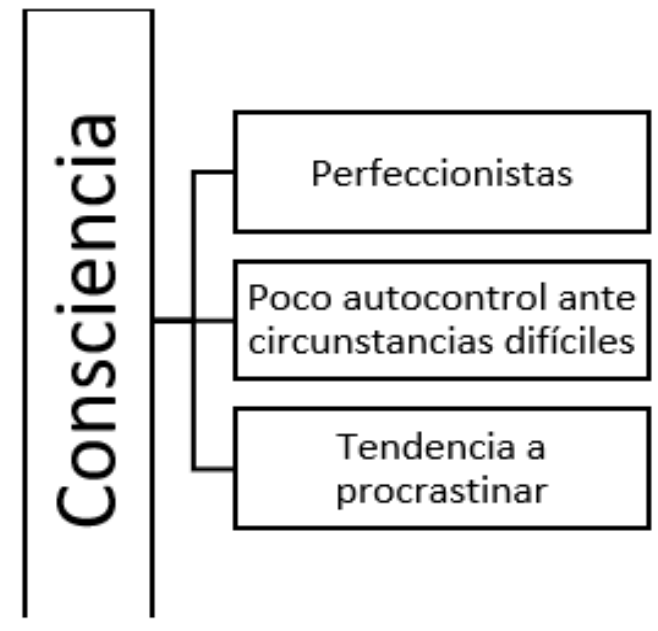

\section{b 2) Motivación}

En cuanto a los intereses personales y académicos, PTEA1, PTEA2, PTEA3, PTEA4 y PTEA5 tienen como prioridad académica terminar la carrera, y del total de los participantes, dos mencionaron su interés por continuar con una maestría; PTEA5 y PTEA6 mencionan como prioridad salir con buen promedio; PTEA6 enumera entre sus expectativas mejorar económicamente: 
Tener un título, seguir estudiando una maestría u otra licenciatura relacionada con el lenguaje (PTEA2). Terminar ya la carrera, y sí pienso estudiar la maestría en Periodismo (PTEA4).

Dedicarme a la animación, ejercer el periodismo. Mis intereses académicos siempre se han reducido a graduarme. [...] utilizar la, la carrera como un medio para obtener ingresos que me permitan dedicarme a otra cosa (PTEA5).

Independizarme [...] vivir sin carencias. [...] terminar la carrera con un buen promedio, dejar una buena impresión como un estudiante que se esfuerza bastante por sus calificaciones (PTEA6).

Cuando platican acerca de qué los apasiona, PTEA2 y PTEA4 coinciden en viajar y aprender algo nuevo; PTEA1 menciona la fotografía; PTEA3, el periodismo; PTEA5 refiere que al menos ahora le gusta analizar películas, y PTEA6 dice que la historia y la literatura.

En otros de los elementos de la subcategoría Motivación, sobre sus valores personales, ponen el respeto como un eje central. Luego, PTEA1, PTEA2, PTEA3 y PTEA5 mencionan la honestidad, y PTEA4, PTEA5 y PTEA6 aluden a la responsabilidad:

La honestidad, el respeto, no violentar a las personas (PTEA1).

Respeto hacia los demás y la educación, la honestidad (PTEA2).

Ser honesto, también responsable, de aceptar las consecuencias de mis actos (PTEA5).

Sobre la motivación personal y académica, los mueve principalmente la profesionalización, ser buenos en su trabajo, superarse a sí mismos (PTEA1, PTEA3, PTEA4 y PTEA5) y mejorar sus condiciones de vida (PTEA6):

Ser buena en mi trabajo [...] mi familia, mis papás [...] me he creado una imagen de cómo me quiero ver a futuro y quién soy (PTEA3).

Un buen trabajo, seguir conociendo, profesionalizando (PTEA4).

Un deseo de superación personal, tengo un sentido de querer mejorar, el querer ser algo mejor que yo, de querer superarme a mí mismo, ser un poco más paciente, comprensivo (PTEA5).

La figura 7 compila las coincidencias encontradas en esta subcategoría. 
Figura 7. Coincidencias en la subcategoría Motivación

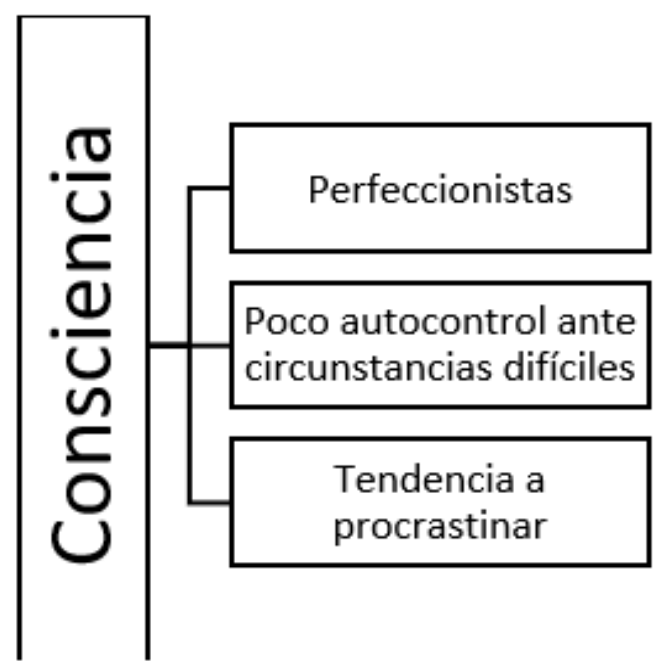

\section{b 3) Voluntad}

Todos los participantes se reconocen como perseverantes y constantemente esforzados para alcanzar sus objetivos:

Me considero muy perseverante (PTEA1).

Sí, pues lo suficiente, depende de lo que se trate, pero sí soy buena en eso (PTA2).

La figura 8 muestra las coincidencias en esta subcategoría.

Figura 8. Cruce de coincidencias de la subcategoría Voluntad

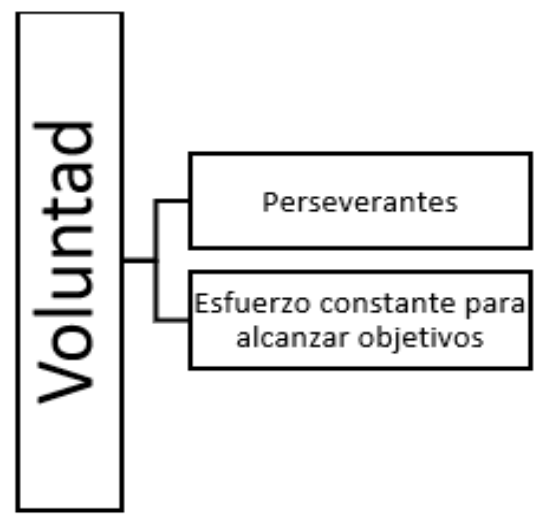




\section{Recursos}

Por último, con respecto a la categoría de Recursos, referente al talento en la redacción, PTEA3, PTEA4, PTEA5 y PTEA6 se consideran muy buenos para redactar:

Sí, creo que sí [...] sé que, si me tomo el tiempo, y me siento, y lo hago con calma, el texto sale muy bien, y me lo han dicho mis profesores, que tiene muy buenas ideas, "los plasmas muy bien" (PTEA3).

Muy bueno, creo, yo diría (PTEA4).

Muy bueno (PTEA6).

PTEA1 y PTEA2 no se consideran buenos para redactar, pero sí con facilidad para ello si cuentan con tiempo:

No me considero buena [...] yo batallo mucho para escribir bajo presión, yo tardo bastante, y para mí es como un ritual, y a veces escribo algo y siento que no me queda a la primera (PTEA1).

No [...] No sé, pues, soy buena en el aspecto de forma, pero en el contenido no, no [...] de forma relativa, yo diría, porque sí puedo escribir, pero también me lleva mucho tiempo, a veces (PTEA2).

En cuestión de talento en la escritura, PTEA3, PTEA4, PTEA5 y PTEA6 se consideran talentosos:

Yo creo que sí, yo creo que sí, y... y haberlo identificado me ayudó a seleccionar la carrera... (PTEA4). Creo que sí, porque creo que, creo que di un, creo que hubo un cambio muy grande de primero a segundo semestre en mi manera de redactar, y mis compañeros también me lo han señalado, de que yo tuve un avance muy, muy grande (PTEA5).

Respecto a qué le atribuyen su facilidad para escribir, PTEA1, PTEA4 y PTEA6 consideran que son muchos factores, entre ellos, el gusto personal, los apoyos de la familia, pares y maestros:

Muchas personas están involucradas, mi mamá desde esa naturalidad de ser muy comunicativa, en las personas con las que trabajaba [...] En la escuela también, mis amigos, uno siempre aprende de todos o hasta con personas que conozco en la calle, yo digo que hasta la música puede considerarse algo como un texto (PTEA1).

Pues creo que ha sido como un conjunto de cosas, o sea, no es como que algo aislado nada más, creo que ha sido, mmm, ha sido el gusto personal, el, el apoyo de la familia, este, los maestros, los amigos (PTEA4).

Pues más al gusto (PTEA6).

PTEA2 y PTEA3 mencionan que se dieron cuenta de que tenían talento para la escritura en el nivel universitario, mientras que PTEA3 y PTEA4 dicen que fue en un concurso. 
Ya estando aquí en la universidad [...] ya estando aquí, que me empezaron a revisar cosas y no había tantas correcciones, fue así como de que: “jah!” (PTEA2).

El cambio a la universidad, sí me bloqueé un poco las primeras semanas [...] vi que si me esforzaba un poco más, podía mejorar, este, fue cuando dije "ok, entonces sí soy buena, sí es lo mío (PTEA3).

Cuando hice el concurso del Quijote [...] y gané (PTEA3).

Yo creo que fue en la preparatoria, después de que presenté el ensayo, eh, de filosofía, que participé en el concurso de ensayo filosófico (PTEA4).

PTEA5 y PTEA6 recuerdan que se dieron cuenta a partir de un trabajo escrito para alguna clase:

Yo creo que, en mi último semestre de bachillerato, fue, porque teníamos que heredar una escritura o algo así [...] entonces me puse a escribir, entonces dejé que las ideas fluyeran [...] yo creo que esa fue la primera ocasión en la que sentí una verdadera facilidad para escribir (PTEA5).

En tercero, recuerdo que hice un trabajo [...] el profesor me felicitó bastante por el escrito (PTEA6).

Al hablar de otros talentos, PTEA1, PTEA3 y PTEA4 se consideran talentosos en la fotografía, y PTEA3 y PTEA4 en la actuación. Para mejorar y desarrollar aún más esos talentos, PTEA1, PTEA3, PTEA4 y PTEA5 coinciden en que los ha ayudado la práctica constante, así como tomar cursos y talleres:

Sí, practicar [...] entonces empecé a tomar talleres, dos talleres aparte con una fotógrafa de Ciudad Juárez, y también empecé a mandar fotografías a concursos (PTEA1).

He tomado clases, he tomado cursos, invierto, eh, si es algo que me gusta, busco invertir y practicar siempre (PTEA3).

Pues, todo el tiempo tratar de, de escribir, o sea, hacerlo [...] o sea, practicarlo todo el tiempo (PTEA4). Me ha servido mucho revisar trabajos de compañeros, yo creo que ese ha sido el ejercicio [...] más periódico que he realizado para expandir mis habilidades de redacción (PTEA5).

La figura 8 muestra las coincidencias en la información analizada de la categoría Recursos. 
Figura 8. Cruce de coincidencias de la categoría de Recursos. Son elementos transversales a los distintos catalizadores ambientales e interpersonales

Figura 8. Cruce de coincidencias de la categoría de Recursos. Son elementos transversales a los

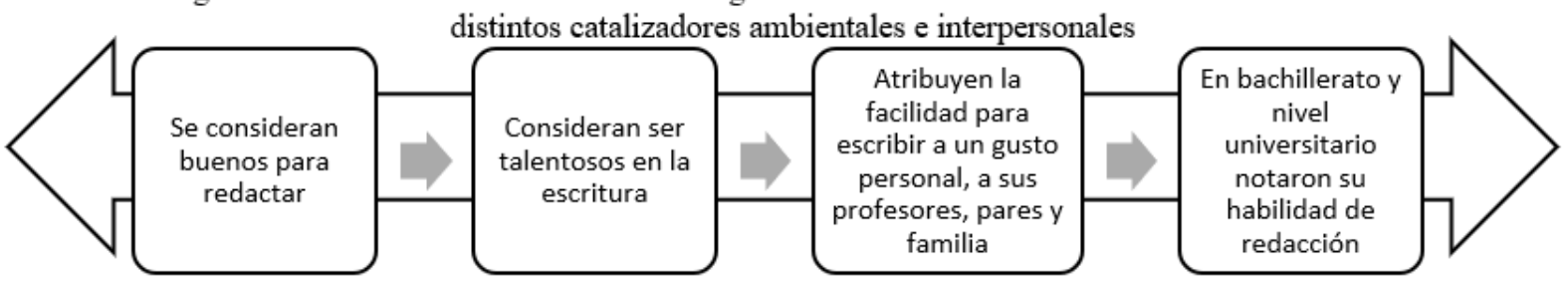

\section{Conclusiones}

Los resultados han arrojado varias coincidencias en los catalizadores, tanto ambientales como intrapersonales, por lo que en estos casos de estudio se encuentran valiosos elementos sobre el talento en la escritura académica de alumnos de Periodismo y del desarrollo del talento en general.

Las coincidencias en los rasgos ambientales refuerzan elementos mencionados por Gagné (2015), en cuanto a que las capacidades naturales (por ejemplo, leer a temprana edad, el gusto por la lectura desde esas primeras etapas y la participación en concursos por tener aptitudes destacadas en la lectura o escritura) y el trabajo constante, bajo diversos catalizadores, permiten el desarrollo del talento, en este caso en la escritura académica.

En referencia a los catalizadores intrapersonales estudiados, los datos sobre al manejo de objetivos remiten a algo similar a lo encontrado por Fletcher y Speirs (2012): en los sujetos talentosos (o sobredotados) el perfeccionismo y su motivación para concretar objetivos están presentes dada su constancia y esfuerzo (perseverancia).

Entre los aspectos que ayudan a la construcción del perfil del talento en la escritura académica de los estudiantes de Periodismo, se encuentra que los individuos (profesores y pares) son parte relevante para desarrollar el talento, pues las figuras de los profesores o mentores, así como el apoyo de los pares, han sido guía y fuente de retroalimentación para los procesos de mejora escritural. Por lo que hace a la familia, es relevante el hecho de que no aparecen datos de tradición redactora o lectora en los casos estudiados. En tanto, los rasgos físicos (como la habilidad en la motricidad fina) y la percepción de sí mismos (perseverantes, responsables, en búsqueda de superación personal y profesional) resultan fundamentales para el proceso de desarrollo de las competencias del talento en la escritura académica.

Con este estudio se colabora el desarrollo de un perfil del talento en la escritura académica, y además podría incidirse en características para un replanteamiento curricular de programas educativos, ya que la primera intención es mostrar qué se identifica como un talento de escri- 
tura en los estudiantes de Periodismo y cuáles son las características que lo evidencian, a partir de la creación de un modelo que bosqueje el perfil desde una perspectiva académica (Pérez, López, González, 2008). Representa un aporte significativo, pues no existe a la fecha un concepto y un perfil para este tipo de talento, menos aún en jóvenes y adultos.

Sin embargo, es importante reconocer que existen limitantes teórico-metodológicas, dado el enfoque, la muestra y el modelo teórico, pues se podría obtener datos con otros componentes si se emplean concepciones distintas de talento (dado el modelo bajo el cual se explique); también, si el enfoque metodológico cambiara o tomara más casos de estudio. Además, la literatura existente sobre el tema en específico es muy poca, incluso en otros idiomas, pues se ha realizado más trabajo sobre lectura y escritura académica, pero no enfocado al talento de dichas áreas, de lo cual dan cuenta investigaciones como las de Carrasco, Fátima, Castro y López (2013), Salazar (2016) y Asencio (2019).

Este planteamiento permite empezar a modelar un plan de formación escolar (e idealmente familiar) para promover un mejor desempeño en la escritura académica de alumnos de Periodismo, ya que la construcción de textos, la habilidad para saber trabajar las ideas y palabras requiere trabajo constante e innovación, pues este desarrollo repercute directamente en la eficacia comunicativa que dicha profesión requiere (Díaz, Catalá, 2015).

Se propone, además, realizar más estudios del tema, sobre el aspecto de los servicios que apoyan como catalizadores, pues no fueron explorados, así como puntualizar varios elementos de los estudiados en los otros catalizadores, con miras a fortalecer el perfil del talento en la escritura académica de estudiantes de Periodismo y, eventualmente, replicarlo y trabajar con otros programas educativos universitarios.

\section{Referencias}

Arcanio, M.; C. Falavigna; P. Soler (2013). Ingreso y desconcierto: ¿Nuevas preguntas y viejas estrategias? Sobre los jóvenes, la relación con el conocimiento y la construcción de subjetividades. Cuadernos de Educación, 11(11), 1-13.

https://revistas.psi.unc.edu.ar/index.php/Cuadernos/article/view/6037

Asencio, M. (2019). La lectura y la escritura académica en educación superior: el taller como estrategia didáctica. Psychology, Society, and Education, 11(2), 205-219.

http://ojs.ual.es/ojs/index.php/psye/article/view/2079

Betancourt, J.; M. Valadez (2012). Cómo propiciar el talento y la creatividad en la escuela. México: Manual Moderno.

Carlino, P. (2003). Alfabetización académica: un cambio necesario, algunas alternativas posibles. Educere, 6(20), 409-420. https://www.redalyc.org/pdf/356/35662008.pdf 
Carrasco, M.; M. Fátima; M. Castro; G. López (2015). Lectura y escritura académica en la educación media superior y superior. Revista Mexicana de Investigación Educativa, 18(57), 349354. http://www.scielo.org.mx/pdf/rmie/v18n57/v18n57a2.pdf

Díaz, B.; M. Catalá (2015). Hablar para escribir. El estilo periodístico. Atenas, 1(29), 67-81. https:// www.redalyc.org/pdf/4780/478047205005.pdf

Escalante-Barreto, C. (2015). La escritura académica como proceso epistémico en la enseñanza del derecho penal. Educación y Educadores, 18(2).

https://www.redalyc.org/pdf/834/83441028003.pdf

Flanagan, A.; V. Arancibia (2005). Talento académico: Un análisis de la identificación de alumnos talentosos efectuada por profesores. Psykhe (Santiago), 14(1), 121-135. https://scielo.conicyt.cl/scielo.php?pid=S0718-22282005000100010\&script=sci arttext

Fletcher, K.; K. Speirs (2012). Research on Perfectionism and Achievement Motivation: Implications for Gifted Students. Psychology in the Schools, 49(7), 668-677. https://www.researchgate.net/publication/230730151 Research on perfectionism and achievement motivation Implications for gifted students

Gagné, F. (2008). Building Gifts into Talents: Brief Overview of the DMGT 2.0. High Ability Studies, 152, 81-89. http://www.eurotalent.org/Gagne DMGT Model.pdf

Gagné, F. (2015). De los genes al talento: la perspectiva DMGT/CMTD. Revista de Educación, 368, 12-39.

http://www.educacionyfp.gob.es/dam/jcr:86553cfc-f5cc-4f8d-ad56-c07e96c21e48/de-los-genes-altalento-pdf.pdf

Galdó, G. (2007). Niños superdotados. Granada: Universidad de Granada.

Gallagher, J.; S. Gallagher (1994). Teaching the Gifted, Child. Boston: Allyn \& Bacon.

Marland, S. (1971). Education of the Gifted and Talented. Washington: Government Printing Office. Pérez, L.; E. López; C. González (2008). La detección del talento lingüístico. Faísca, 13(15), 124159. https://dialnet.unirioja.es/servlet/tesis?codigo $=144244$

Reig, L.; S. Reig (2013). Cómo despertar el talento de los niños. España: Toromítico.

Roldán, C.; A. Vázquez; A. Rivarosa (2011). Mirar la escritura en la educación superior como un prisma. Revista lberoamericana de Educación, 55(3), 1-13. https://www.researchgate.net/profile/Alcira Rivarosa/publication/282656197 4010Roldan- OEl2011/links/5616548808ae37cfe40902fc/4010Roldan-OEI2011.pdf

Salazar, C. (2016). La escritura académica universitaria: diferentes perspectivas de estudio. Diálogos sobre Educación, 11(6), 1-21.

http://dialogossobreeducacion.cucsh.udg.mx/index.php/DSE/article/view/275

Shore, B.; L. Kanevsky (1993). Thinking processes: Being and Becoming Gifted. International Handbook of Research and Development of Giftedness and Talent, 1, 133-147.

https://www.researchgate.net/profile/Bruce Shore2/publication/232604665 Thinking processes Being and becoming gifted/links/56ef89ab08aed17d09f87a94.pdf 
Universidad Autónoma de Ciudad Juárez (s.f. a). Perfil de ingreso de la Licenciatura en Periodismo. http://www.uacj.mx/ICSA/Humanidades/LP/Paginas/default.aspx

Universidad Autónoma de Ciudad Juárez (s.f. b). Perfil de egreso de la Licenciatura en Periodismo. http://www.uacj.mx/ICSA/Humanidades/LP/Paginas/default.aspx

Universidad Autónoma de Ciudad Juárez (s.f. c). Objetivos del programa de Periodismo. http:// www.uacj.mx/ICSA/Humanidades/LP/Paginas/default.aspx 